Enfermagem Brasil 2016;15(3):131-6

\title{
ARTIGO ORIGINAL \\ Programa saúde na escola: reflexões sobre a prática
}

Milla Pauline da Silva Ferreira*, Milanda dos Santos Silva*, Anselmo Araújo Oliveira*, Aline Batista Matos*, Tereza Cristina Costa Dantas, M.Sc. ${ }^{* *}$, Naluse Anne Silva Coutinho ${ }^{* * *}$, André Henrique do Vale de Almeida, M.Sc. ${ }^{* * \star}$, Magno Conceição das Merces, M.Sc. ${ }^{* \star \star *}$

${ }^{*}$ Discente do Curso de Enfermagem, Universidade Estadual de Feira de Santana (UEFS), Feira de Santana/BA, ${ }^{* *}$ Enfermeira, Docente do Curso de Enfermagem da UEFS, Feira de Santana/BA, ${ }^{* * *}$ Enfermeira, Prefeitura Municipal de Feira de Santana, Feira de Santana/BA, ${ }^{* * * *}$ Enfermeiro, Docente, Curso de Enfermagem da UEFS, Feira de Santana/BA, ${ }^{* * * * B i o ́ l o g o, ~ E n f e r m e i r o, ~ P r o f e s s o r ~ A u x i l i a r ~ A ~ d a ~ U n i v e r s i d a d e ~ d o ~ E s t a d o ~ d a ~ B a h i a ~(U N E B), ~}$ Doutorando em Enfermagem pela Universidade do Estado do Rio de Janeiro (UERJ)

Recebido em 18 de março de 2015; aceito em 16 de junho de 2015.

Endereço para correspondência: Magno Conceição das Merces, Rua Campo Florido, 204, Mar da Tranquilidade, Feira de Santana BA, E-mail: mmerces@uneb.br, milandasilva@hotmail.com

\section{Resumo}

O estudo tem como objetivo relatar a experiência de estudantes do curso de enfermagem da Universidade Estadual de Feira de Santana (UEFS) acerca da realização do Programa Saúde na Escola (PSE) com adolescentes de um colégio estadual do município. A realização do PSE ocorreu em três etapas: etapa de planejamento, com reuniões com a equipe da Unidade de Saúde da Família e com a direção da escola; execução, com a aferição das medidas antropométricas dos alunos, avaliação da acuidade visual e vacinação; e avaliação, com o levantamento de possibilidades e limites do PSE. Dentre as possibilidades encontradas, destacou-se o apoio do colégio disponibilizando horários e estrutura física. Como limites, aponta-se a adesão parcial dos alunos e a sobrecarga de trabalho da enfermeira dispondo de pouco tempo para realizar o PSE. Faz-se necessário ainda maior envolvimento entre todos os envolvidos no processo, já que resulta em ganhos tanto para os adolescentes quanto para a equipe.

Palavras-chave: saúde escolar, promoção da saúde, Enfermagem, saúde da família.

\section{Abstract \\ Health program in school: reflections on practice}

This study aimed to report a nursing students experience of University of Feira de Santana (UEFS) concerning Health Program in School (HPS) performance with adolescents of a state school in the city of Feira de Santana, Bahia. The HPS was carried out in three stages: planning stage, with meetings with the Family Health Unit team and the school board; implementation with anthropometric measurements of students, assessment of visual acuity and vaccination; and evaluation, with a survey of possibilities and limits of the HPS. Among the possibilities found, school support providing schedules and physical structure stood out. As limits, we point out partial participation of students, and nursing workload with little time to perform the HPS. We observed that more participation is needed among all involved in the process, since it results in gains for both the adolescents and the team.

Key-words: school health, health promotion, nursing, family health.

\section{Resumen}

\section{Programa de salud en la escuela: reflexiones sobre la práctica}

El estudio tiene como objetivo relatar la experiencia de estudiantes del curso de enfermería de la Universidad Estadual de Feira de Santana (UEFS), acerca de la ejecución del Programa de Salud en la Escuela (PSE) con adolescentes de una escuela pública de la ciudad de Feira de Santana, Bahia. El proceso de realización del PSE constó de tres etapas: la etapa de planificación, con reuniones con el equipo de la Unidad de Salud de la Familia y la dirección de la escuela; la toma de medidas antropométricas de los alumnos, la evaluación de agudeza visual y la vacunación; y evaluación, con un estudio de las posibilidades y límites del PSE. 
Entre las posibilidades encontradas, se destacó el apoyo de la universidad poniendo disponibles horarios y estructura física. Como límites señalaron adhesión parcial de los estudiantes y la sobrecarga de trabajo de la enfermera con poco tiempo para realizar el PSE. Es necesaria una mayor participación de todos los involucrados en el proceso, ya que resulta en beneficios tanto para los adolescentes como para el equipo.

Palabras-clave: salud escolar, promoción de la salud, Enfermería, salud de la familia.

Com a instituição do Programa Saúde na Escola (PSE), em 2007, a escola tornou-se um locus para ações de promoção da saúde para crianças, adolescentes e jovens adultos de modo mais concreto. Os sistemas de saúde e educação avançaram na medida em que passaram a atuar em conjunto, partindo do pressuposto de que as ações de saúde devem ser desenvolvidas de forma intersetorial. Desse modo, quando uma ou mais áreas estão envolvidas num trabalho em prol de objetivos comuns, são capazes de resultar em benefícios para todos [1].

A Educação e a Saúde, historicamente, possuíam maior proximidade, especialmente no que diz respeito às políticas públicas de saúde, já que estavam fundamentados em princípios, como a universalização, ou seja, todos tinham direito a saúde e também a educação [1]. Na década de 50, ambos faziam parte de um único ministério, o Ministério da Educação e Saúde, sendo posteriormente dividido em dois, formando o Ministério da Saúde e o Ministério da Educação.

Na prática, a responsabilização pela promoção da saúde acabou restrita, em sua maior parte, ao Ministério da Saúde. A Atenção Básica é uma das portas de entrada dos serviços de saúde, apresentando a filosofia de promover a qualidade de vida e o bem-estar individual e coletivo por meio de ações e serviços de promoção, proteção e recuperação da saúde. Nesse sentido, a Estratégia de Saúde da Família (ESF) conhecida anteriormente como Programa Saúde da Família (PSF), instituído em 1994, prioriza as ações de proteção e promoção da saúde, através do atendimento prestado tanto na unidade básica de saúde como nos demais espaços da comunidade [2-3]. Outrossim, a escola já faz parte dos campos de atuação das equipes de PSF, mesmo que de forma implícita.

O PSE é fruto do Decreto Presidencial oㅜ 6.286/2007 gestado de uma política intersetorial do Ministério da Saúde e do Ministério da Educação e entre as suas diretrizes destacam-se a descentralização e respeito à autonomia federativa; integração e articulação das redes públicas de ensino e de saúde; territorialidade; interdisciplinaridade e intersetorialidade; cuidado ao longo do tempo; controle social e monitoramento, entre outros [4]. O principal objetivo do programa em discussão é contribuir para a formação integral dos estudantes por meio de ações de promoção, prevenção e atenção à saúde, com vistas ao enfrentamento das vulnerabilidades que comprometem o pleno desenvolvimento de crianças e jovens da rede pública de ensino. Destaca-se como uma estratégia de integração da saúde e educação para o desenvolvimento da cidadania e da qualificação das políticas públicas brasileiras [5].

As ações preconizadas pelos Ministérios da Saúde e de Educação dentro do PSE são divididas em três componentes, a saber: as ações do Componente I, que correspondem a "Avaliação Clínica e Psicossocial" dos discentes, envolvendo a avaliação antropométrica, atualização do calendário vacinal, detecção precoce de agravos à saúde, avaliação oftalmológica, avaliação nutricional, avaliação da saúde bucal, avaliação psicossocial, entre outras. Esta primeira etapa assume um caráter de triagem, haja vista que ao ser identificado algum problema clínico e/ou psicossocial o aluno deverá ser encaminhado para a Unidade de Saúde da Família (USF), a fim de obter um acompanhamento individualizado [6]. Todo e qualquer encaminhamento deve ser articulado com a equipe da escola, para introduzi-la como corresponsável pela saúde dos seus discentes.

Em sequência, o Componente II, intitulado como "Promoção e Prevenção à Saúde", visa identificar os fatores que colocam a saúde das crianças e adolescentes em risco e atuar sobre eles visando a sua redução e eliminação. Acredita-se que a Educação em Saúde é um processo fundamental para alcançar este objetivo, o programa prioriza alguns temas a serem abordados dentro da sala de aula, entre eles: segurança alimentar e promoção da alimentação saudável, promoção das práticas corporais e atividade física nas escolas, educação para a saúde sexual, prevenção ao uso de álcool e tabaco e outras drogas, promoção da cultura de 
paz e prevenção das violências, e promoção da saúde ambiental e desenvolvimento sustentável.

O Componente III inclui a "Formação de grupos" para manutenção dos objetivos do programa. Exemplos: Grupo de Trabalho Intersetorial (GTI); formação de Jovens Protagonistas para o PSE; curso de Prevenção do Uso de Drogas para Educadores de Escolas Públicas e a Rede Universidade Aberta do Brasil [6]. Diante do extenso leque de ações supracitadas, fica clara a necessidade da articulação direta entre todos os profissionais da USF, incluindo enfermeiros, agentes comunitários de saúde (ACS), técnicos em enfermagem, dentistas e médicos. Ademais, o desenvolvimento das ações também depende da parceria com a equipe do Núcleo de Apoio à Saúde da Família (NASF).

Pontua-se que as ações do PSE são de relevência para os estudantes envolvidos no processo, profissionais da USF e para a comunidade acadêmica que desenvolve práticas nas unidades, haja vista que, a partir do vínculo entre saúde, educação e comunidade, é possível garantir a promoção da saúde, prevenção de agravos e melhorias na qualidade de vida da população, além de solidificar a relação entre equipe de saúde e comunidade. A realização deste estudo justifica-se devido a necessidade de expor à comunidade científica, aos profissionais e gestores sobre a relevância do PSE realizado pela equipe da USF, esclarecendo o funcionamento do programa, os limites e possibilidades encontradas.

Desta forma, formulou-se a seguinte questão norteadora: Quais as percepções, nuances e dificuldades vivenciadas por estudantes do curso de enfermagem na prática do PSE?

Neste sentido, este estudo tem como objetivo relatar a experiência de estudantes do curso de enfermagem da Universidade Estadual de Feira de Santana (UEFS) acerca da realização do PSE com adolescentes de um colégio estadual do município.

Material e métodos

Trata-se de um relato de experiência de estudantes do 9o semestre do curso de graduação em enfermagem da Universidade Estadual de Feira de Santana que atuaram no PSE realizado com um grupo de estudantes adolescentes de um colégio público, de Feira de Santana na Bahia, no período de maio a junho de 2014.

O colégio funciona desde 18 de março de 1985 e atualmente apresenta 19 salas de aula e cerca de 2000 alunos, distribuídos em três turnos, manhã, tarde e noite com três modalidades de ensino: Fundamental, Médio e Educação de Jovens e Adultos (EJA).

O público-alvo das ações do PSE realizadas foram 154 adolescentes dos 464 alunos do Ensino Médio, o que corresponde a 33,9\% do total. Utilizaram-se como critérios de inclusão aqueles que estavam entre a faixa etária de 10 a 24 anos de idade, de acordo com as definições da Organização Mundial da Saúde (OMS) e do Estatuto da Criança e do Adolescente (ECA) [7,8].

Para elaboração deste artigo, foram utilizadas as seguintes técnicas e instrumentos: elaboração do plano de ações para o PSE; relatório de realização do PSE; fichas de atividade coletiva; participação nas atividades: avaliação nutricional e da acuidade visual dos adolescentes.

Resultados

As atividades do PSE foram desenvolvidas pela equipe da UEFS (professor da disciplina de Estágio Supervisionado I do curso de enfermagem e quatro estudantes), equipe de saúde da USF (enfermeira, ACS, técnica de enfermagem, dentista e auxiliar de consultório dentário) e equipe do NASF, através de visitas ao colégio, desenvolvendo as fases de planejamento, execução e avaliação do processo.

Durante a etapa de planejamento, foi feita uma reunião com a equipe na USF Feira VI, com enfermeira, estudantes de enfermagem, ACS e equipe do Núcleo de Apoio à Saúde da Família (NASF) para definição de data, local, horário e programação do PSE no colégio.

Posteriormente, houve uma reunião com a diretora da escola para planejar o PSE, confirmar data, local, horário e programação, solicitar quantitativo de alunos matriculados do ensino médio e reforçar a necessidade dos alunos trazerem cartão SUS, cartão de vacina e certidão de nascimento ou identidade, pois estes seriam necessários para a avaliação do PSE.

Foram elaborados também avisos para serem entregues aos alunos a fim de informálos sobre as ações que seriam realizadas pela equipe e pelos estudantes de enfermagem, 
além da necessidade de levar os documentos preconizados pelo PSE. Esses avisos foram entregues a diretora da escola para serem distribuídos aos alunos.

Durante a etapa de execução, realizou-se no colégio breve apresentação da proposta do PSE ao corpo docente e distribuição da equipe no colégio para executar as ações referentes ao componente I do PSE. Em uma sala ficou a técnica de enfermagem e um estudante de enfermagem para atualização dos cartões de vacina e aplicação de imunobiológicos (tríplice viral, $\mathrm{dT}$, hepatite $\mathrm{B}$, febre amarela, influenza). Em outra sala, a enfermeira, os demais estudantes e os ACS realizaram as medidas antropométricas dos alunos, avaliação da acuidade visual utilizando o teste de Snellen e preenchendo os formulários para inclusão no Cadastro Nacional de Saúde (para os alunos que não possuíam o cartão SUS).

$\mathrm{Na}$ etapa de avaliação a equipe foi reunida para avaliar as ações executadas no colégio. Foram levantadas as possibilidades $e$ os limites encontrados durante a operacionalização do PSE e desenvolvidas estratégias para superar as dificuldades. Durante esta etapa, percebeu-se que do total de 464 alunos que compõem o ensino médio, apenas 154 adolescentes foram avaliados e somente 32 levaram o cartão SUS, evidenciando a necessidade de avaliação das ações do PSE com a finalidade de identificar os fatores que podem interferir na realização das atividades.

Identificou-se também a necessidade da equipe continuar o processo do PSE, realizando as ações do componente II (promoção e prevenção à saúde) e componente III (formação de grupos) para alcançar todos os objetivos do programa.

Discussão

A realização do PSE foi de fundamental importância para os adolescentes do colégio, pois proporcionou ganhos à saúde dos mesmos, já que foi um momento de avaliação do peso, altura e acuidade visual, associado a esclarecimentos sobre diversas dúvidas sobre o peso ideal, alimentação saudável, adolescência, dentre outros.

Em relação ao teste da acuidade visual, procurou-se durante a realização do teste, discutir com os alunos sobre os problemas da visão e a importância do teste, já que através dele poderiam ser detectadas alterações da acuidade visual e, assim, eles poderiam receber tratamento adequado.

Ademais, a prática do PSE possibilitou aos estudantes de enfermagem, durante o estágio, uma oportunidade de aprendizado, no sentido da promoção da saúde, além de ter proporcionado a realização de atividades educativas em saúde. Para a equipe de saúde da USF foi um momento de levar até a escola serviços de promoção, proteção e recuperação da saúde, instituindo a escola como campo de atuação da equipe.

Durante o processo do PSE, verificaram-se os seguintes aspectos positivos: o apoio do colégio para a realização do PSE disponibilizando horários e estrutura física para que pudessem ser realizadas as ações; a adesão dos alunos para as avaliações; a compreensão dos professores do colégio quando foi preciso retornar a escola para finalizar as avaliações, cedendo um período do horário de aula; e a disponibilidade da enfermeira, da professora e estudantes de enfermagem, dos ACS e da equipe do NASF.

O PSE permitiu que fosse identificada a realização de importantes atividades relacionadas com a questão da saúde na escola. A realização do "Dia $D$ da Família" é um exemplo, pois o colégio abriu as portas para a comunidade e ofereceu serviços como aferição de pressão, teste de glicemia, tipagem sanguínea, vacinação, atendimento médico, palestras sobre acidentes domésticos, massoterapia, cuidados com o cabelo, balcão de justiça e cidadania e oficinas de artes. Percebemos que nos dias em que há eventos de grande porte como o supracitado, as atividades do PSE devem ser direcionadas para educação em saúde, visto que toda a comunidade pode participar.

A equipe também teve a oportunidade de presenciar o dia das provas da Olimpíada Brasileira de Matemática, que mobilizou os alunos para a realização de provas que testam o conhecimento em matemática. As atividades do PSE foram compartilhadas com a realização das provas da olimpíada, contudo, com o apoio da diretora as ações do PSE não prejudicaram a mesma.

A reflexão sobre o processo do PSE aponta que se deve evitar marcar os procedimentos de aferição nos dias de grandes eventos no colégio, pois provocou a dispersão dos alunos, esquecimento dos documentos necessários, dificultando a realização das ações previstas. 
Um dos limites para a realização do PSE foi a adesão parcial dos alunos. Por estarem na adolescência, identificada como uma fase complexa, período de transição entre a infância e a vida adulta, e caracterizada pelos impulsos tanto do desenvolvimento físico quanto mental, emocional, sexual e social [9] fez com que muitos ficassem resistentes a passarem pelos testes. Às vezes acima ou abaixo do peso, baixo ou alto demais, ficavam envergonhados na frente dos colegas ou se recusavam até sem motivos evidentes.

Outro aspecto analisado foi a disponibilidade da equipe para atuar no PSE. Evidenciouse que a enfermeira apresenta sobrecarga de trabalho na USF, dispondo de pouco tempo para realizar o PSE. Há somente um enfermeiro na USF para realizar todas as consultas (pré-natal, preventivo, puericultura, entre outros); supervisionar os técnicos de enfermagem (na vacinação, curativos, esterilização de material); verificar e orientar as ações dos ACS; articular com a Secretaria Municipal de Saúde (reuniões, ofícios), entre outras atividades [2].

Verificou-se que a realização das atividades do PSE, apenas uma vez por semana, acarreta demora em concluí-las. Outro ponto a ser considerado como entrave para 0 andamento do programa é o déficit na quantidade de materiais, como fita métrica, balanças e fichas para inclusão no cartão SUS.

Destacam-se como a maior dificuldade encontrada pela equipe, a baixa resolutividade dos problemas detectados, a inexistência da contrarreferência e o limitado número de vagas para as especialidades médicas necessitadas pela população em questão. Entendemos que sem a devida articulação entre a escola e a saúde, o futuro do PSE ficará comprometido.

Entretanto, apesar das dificuldades encontradas durante a realização do PSE, a equipe conseguiu realizar o planejamento prévio, a elaboração de projetos e relatórios e o trabalho em equipe, garantindo, assim, o sucesso das atividades.

\section{Conclusão}

Operacionalizar o PSE foi fundamental para os alunos do colégio, pois contribuiu para o controle e/ou erradicação das doenças infectocontagiosas e imunopreveníveis, através da imunização sistemática dos adolescentes e permitiu ações de educação em saúde (alimentação saudável e da prática de exercício físico para manter o peso adequado), além de informações sobre os serviços de atenção à saúde que são oferecidos para crianças e adolescentes na USF.

A realização do PSE contribui ainda para a formação profissional dos estudantes de enfermagem na medida em que permite: atuação em ações de promoção, prevenção e atenção à saúde dos adolescentes; articulação com a equipe de saúde da USF; estreitamento do vínculo entre a unidade de saúde e a comunidade; conhecimento da realidade e do perfil de saúde-doença dos adolescentes da sua área de abrangência, proporcionando, assim, um momento de aprendizado para todos os envolvidos neste processo.

Esta experiência no PSE mostrou que a sustentabilidade e qualidade deste trabalho dependem de todos os participantes envolvidos. Para obter resultados positivos na realização das ações, faz-se necessário maior envolvimento da equipe do NASF, dos profissionais que compõem a USF, da equipe da escola e demais participantes, em todas as atividades propostas pelo projeto. Entendemos que o PSE não deve ser algo pontual, mas sim ações sistemáticas que visam aproximar os escolares à saúde pública, proporcionando qualidade de vida para os assistidos.

\section{Referências}

1. Brasil. Ministério da Saúde. Secretaria de Atenção à Saúde. Departamento de Atenção Básica. Saúde na escola. Brasília: Ministério da Saúde; 2009.

2. Brasil. Ministério da Saúde. Secretaria de Atenção à Saúde. Departamento de Atenção Básica. Política Nacional de Atenção Básica. Brasília: Ministério da Saúde; 2006. 60 p.

3. Souza RA, Carvalho AM. Programa de Saúde da Família e qualidade de vida: um olhar da Psicologia. Estudos de Psicologia 2003;8(3):515-23.

4. Brasil. Presidência da República. Decreto $n^{\circ}=6.286$, de 5 de dezembro de 2007. Institui o Programa Saúde na Escola - PSE, e dá outras providências. Diário Oficial da União, Poder Executivo, Brasília, DF, 5 dez. 2007. 
5. Conceição J. Programa Saúde na Escola. Publicado em: 11/04/2012. [citado 2014 junho 10]. Disponível em URL: http://www.artigonal.com/educacao-artigos/programasaude-na-escola-5818235.html

6. Brasil. Ministério da Saúde. Secretaria de Atenção à Saúde. Departamento de Atenção Básica. Passo a passo PSE: Programa Saúde na Escola: tecendo caminhos da intersetorialidade. Brasília: Ministério da Saúde; 2011.46 p.

7. Brasil. Estatuto da criança e do adolescente. $7^{\mathrm{a}}$ ed. Brasília: Câmara dos Deputados, Edições Câmara; 2010. 225 p.

8. World Health Organization. Young People's Health - a Challenge for Society. Report of a WHO Study Group on Young People and Health for All. Technical Report Series 731. Geneva: WHO; 1986.

9. Eisenstein E. Adolescência: definições, conceitos e critérios. Adolescência \& Saúde $2005 ; 2(2)$. 